\title{
Disruption of the perineuronal net in the hippocampus or medial prefrontal cortex impairs fear conditioning
}

\author{
Michael J. Hylin, ${ }^{1}$ Sara A. Orsi, ${ }^{1}$ Anthony N. Moore, ${ }^{1}$ and Pramod K. Dash ${ }^{1,2,3}$ \\ ${ }^{1}$ Department of Neurobiology and Anatomy, ${ }^{2}$ Department of Neurosurgery, The University of Texas Medical School, Houston, \\ Texas 77225 , USA
}

\begin{abstract}
The perineuronal net (PNN) surrounds neurons in the central nervous system and is thought to regulate developmental plasticity. A few studies have shown an involvement of the PNN in hippocampal plasticity and memory storage in adult animals. In addition to the hippocampus, plasticity in the medial prefrontal cortex (mPFC) has been demonstrated to be critical for the storage of long-term memory, particularly memories for temporally separated events. In the present study, we examined the role of PNN in the acquisition and retention of memories for trace (in which the conditioned and unconditioned stimuli are temporally separated) and delayed (in which the conditioned and unconditioned stimuli overlap) fear conditioning in both the hippocampus and the mPFC. Consistent with a role for the hippocampus in memory storage in both delayed and trace fear conditioning, removal of hippocampal PNN disrupted contextual and trace fear memory. Disruption of the PNN in the mPFC impaired long-term trace and conditioned stimulus (CS)-elicited fear memory in the trace fear conditioning task. Interestingly, CS-elicited fear memory was also impaired when a delayed fear conditioning paradigm was used. These findings further support a role for the PNN in neural plasticity and implicate PNN-regulated plasticity in neocortical memory storage.
\end{abstract}

The extracellular matrix (ECM) that surrounds neurons (also referred to as the perineuronal net or PNN) has been proposed to play an important role in synaptic stabilization and plasticity during development (Pizzorusso et al. 2002, 2006; Nowicka et al. 2009). The backbone of the PNN is hyaluronic acid (HA; also known as a hyaluronan or hyaluronate) to which chondroitin sulfate proteoglycans (CSPGs) are covalently linked. It has been reported that the primary sensory and motor areas contain considerably more PNN than secondary or higher order association areas in the adult brain (Hockfield and McKay 1983; Hendry et al. 1988; McGuire et al. 1989; Bruckner et al. 1994, 1999, 2008; Hausen et al. 1996). As secondary and association regions are thought to be more plastic than primary sensory and motor areas, it has been suggested that the PNN may also play a role in regulating synaptic plasticity in the adult brain.

A role for the PNN in learning and memory in the adult brain is beginning to emerge (Gogolla et al. 2009; Kochlamazashvili et al. 2010). For example, enzymatic degradation of CSPGs, or removal of the ECM protein tenascin-R, reduces long-term potentiation (LTP) and long-term depression (LTD) in hippocampal slice preparations (Saghatelyan et al. 2000; Bukalo et al. 2001). Furthermore, degradation of HA in the hippocampus impairs retention of contextual fear memory (Kochlamazashvili et al. 2010). Although these and other studies have begun to identify a role for hippocampal PNN, the role of the PNN in memory storage in the neocortex, where it is found to be primarily associated with inhibitory neurons (Alpár et al. 2006), has yet to be fully elucidated.

Previous studies have shown that the rodent medial prefrontal cortex (mPFC), a higher order association area in the neocortex, is necessary for the acquisition of trace conditioning (Kronforst-Collins and Disterhoft 1998; Weible et al. 2000; McLaughlin et al. 2002). For example, Kronforst-Collins and

\footnotetext{
${ }^{3}$ Corresponding author

E-mail p.dash@uth.tmc.edu

Article is online at http://www.learnmem.org/cgi/doi/10.1101/lm.030197.112.
}

Disterhoft (1998) demonstrated that lesions of the rabbit mPFC impair eyeblink conditioning when the conditioned and unconditioned stimuli were separated by a trace period, but not when the stimuli overlapped as occurs during delayed conditioning. In addition to memory acquisition, plasticity in both $\mathrm{mPFC}$ and the hippocampus is required for the storage of trace fear memory (McLaughlin et al. 2002; Dash et al. 2004). We therefore used a combination of chondroitinase plus hyaluronidase to degrade the PNN in the mPFC or dorsal hippocampus (dHPC), and investigated the consequences of its removal in the acquisition and retention of trace and delayed fear.

\section{Results}

Intra-parenchymal infusion of chondroitinase plus hyaluronidase disrupts the perineuronal net

Prior to initiation of learning and memory testing, we examined if the combination of chondroitinase + hyaluronidase would result in removal of the PNN. A group of rats $(n=4)$ was given targeted infusions of chondroitinase + hyaluronidase into one hippocampus, while an equal volume of vehicle was simultaneously infused into the contralateral hippocampus. Animals were killed $3 \mathrm{~h}$ later and $40-\mu \mathrm{m}$ thick brain sections were prepared on a cryostat for immunohistochemical examination of CSPGs using biotinylated Wisteria floribunda agglutinin (WFA). Biotinylated WFA is a plant lectin that binds to CSPGs and can be visualized using fluorescently labeled streptavidin (Hartig et al. 1992; Gogolla et al. 2009). In vehicle-treated hippocampi, we observed a greater presence of PNN in the CA3/CA2 subfields, a moderate amount in the dentate gyrus, and a near absence of net in the CA1 subfield (Fig. 1A). Chondroitinase + hyaluronidase infusion resulted in a loss of WFA binding throughout the dHPC. Combined treatment did not alter NeuN immunoreactivity, indicating that the loss of PNN did not cause overt neuronal loss (Fig. 1B), nor did it affect parvalbumin immunoreactivity, a marker for inhibitory neurons (Fig. 1C). 
A

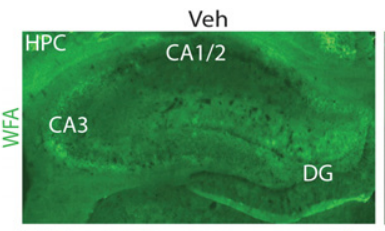

B

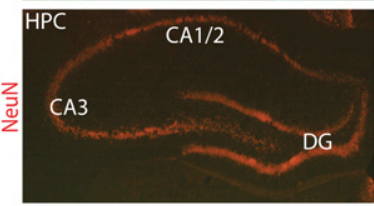

C

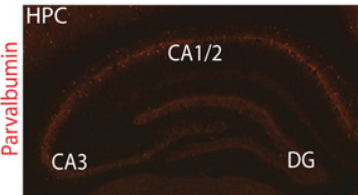

D

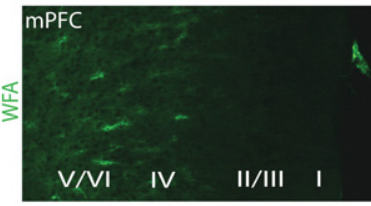

E

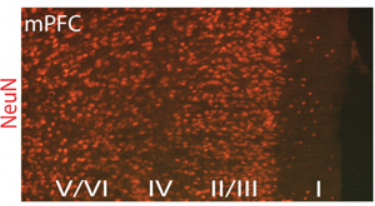

$\mathbf{F}$

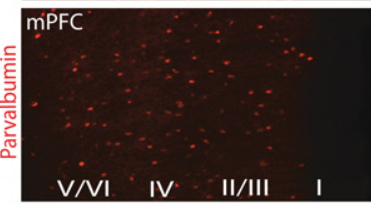

G
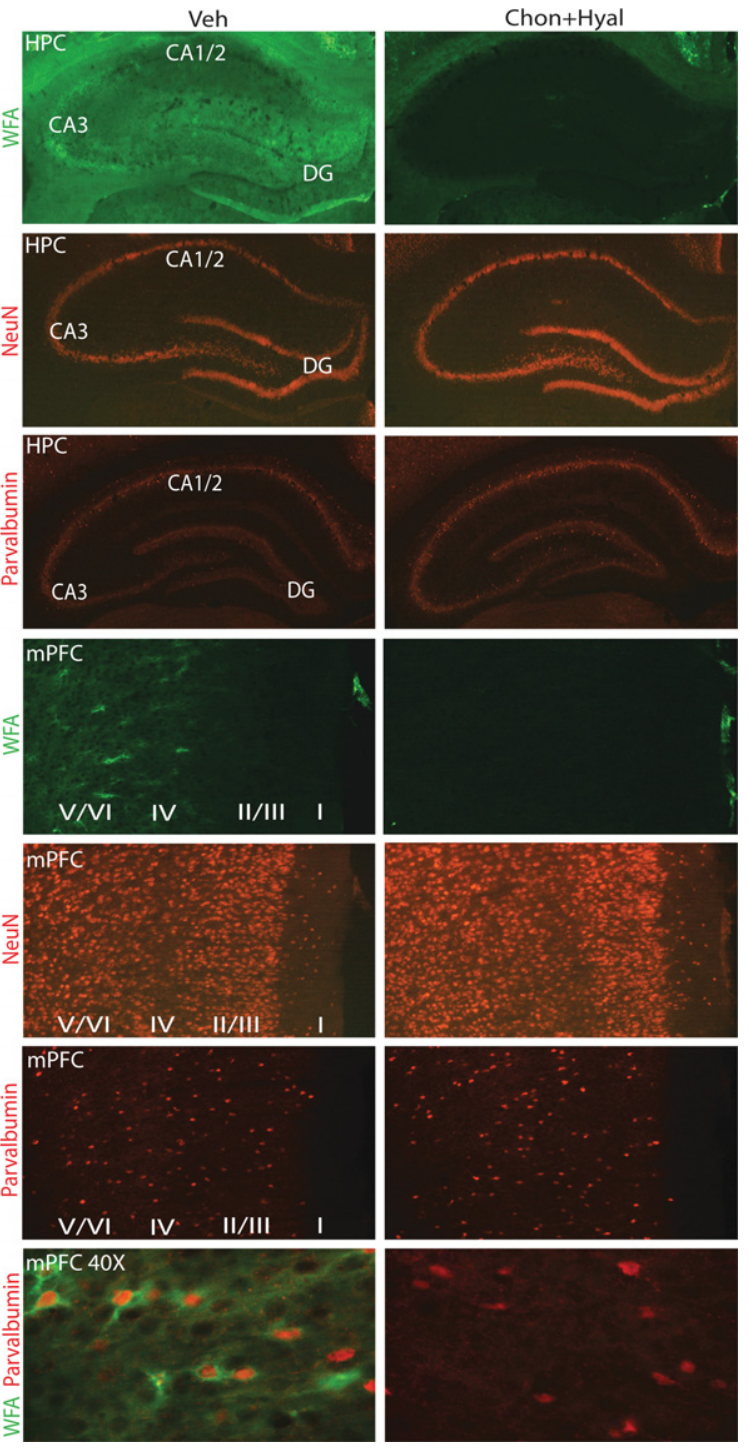

Figure 1. Intra-hippocampal or intra-mPFC infusion of chondroitinase + hyaluronidase degrades PNNs. $(A)$ Representative photomicrographs showing that infusion of chondroitinase + hyaluronidase effectively removes PNNs in the CA1, CA3, and dentate gyrus (DG) subfields of the hippocampus as indicated by loss of WFA staining. (B) Enzyme infusion did not cause any overt neuronal loss as demonstrated by NeuN immunohistochemistry. (C) Combined treatment of chondroitinase + hyaluronidase does not result in the overt loss of parvalbuminpositive neurons. (D) WFA staining revealed that combined treatment with chondroitinase + hyaluronidase completely removed PNNs from the mPFC assessed 3-h post-infusion. (E) Staining for NeuN shows that infusion of chondroitinase + hyaluronidase does not cause visible loss of mPFC neurons. $(F)$ Combined treatment of chondroitinase + hyaluronidase does not result in the overt loss of parvalbumin-immunopositive mPFC neurons. (G) PNNs in the mPFC surround parvalbumin-positive inhibitory neurons.

We next tested the efficacy of chondroitinase + hyaluronidase to remove mPFC PNN by infusing these enzymes into the ipsilateral $\mathrm{mPFC}$ and an equal volume of vehicle was simultaneously infused into the contralateral mPFC. Figure 1D shows that in the vehicle-infused side, PNNs were predominately found in the deep cortical layers (layers IV-VI) of the mPFC, with limited staining observed in layers II and III. Enzyme infusion effectively removed the PNNs as indicated by the loss of WFA immunoreactivity at 3-h post-infusion. As seen in the hippocampus, infusions had no overt effect on mPFC neuronal survival as indicated by NeuN (Fig. 1E) and parvalbumin immunohistochemistry (Fig. 1F). Consistent with that reported for other neocortical areas, the high magnification images shown in Figure 1G indicate that the PNN in the mPFC exists as a network and is more intense surrounding parvalbumin-positive inhibitory neurons.

\section{Intra-hippocampal chondroitinase + hyaluronidase infusion impairs long-term contextual fear memory} Rats received bilateral intra-hippocampal infusions of chondroitinase + hyaluronidase $(n=6)$ or vehicle $(n=8) 3 \mathrm{~h}$ prior to fear conditioning training (Fig. 2A). Figure 2B illustrates that removal of the PNN in the hippocampus prior to training did not influence acquisition of delayed fear $\left(F_{(1,13)}=0.60, P=\right.$ 0.45 ). When tested $48 \mathrm{~h}$ later, contextual (vehicle, $62.00 \% \pm$ $6.39 \%$; chondroitinase + hyaluronidase, $16.33 \% \pm 5.21 \%, P=$ 0.001) (Fig. 2C) but not cue-elicited (vehicle, $82.63 \% \pm 8.43 \%$; chondroitinase + hyaluronidase, $63.33 \% \pm 17.25 \%, P=0.66)$ fear

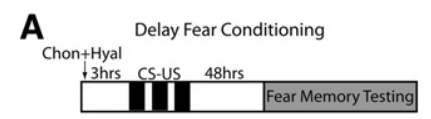

B
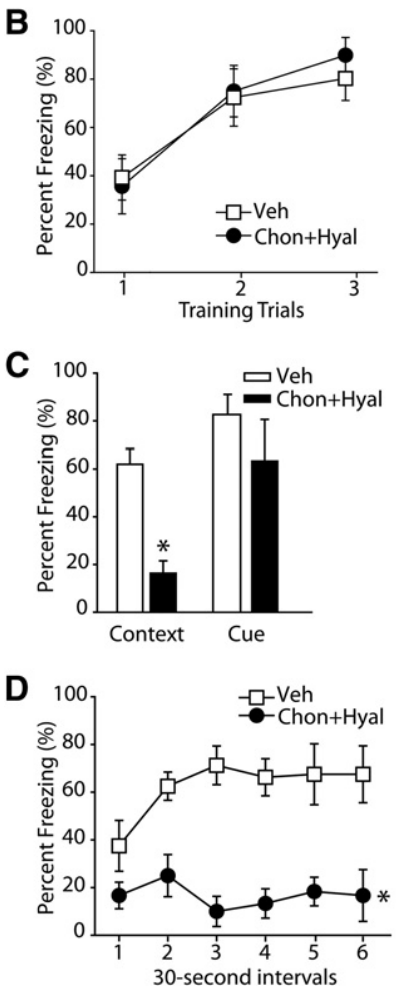

Figure 2. Infusion of chondroitinase (Chon) + hyaluronidase (Hyal) into the dHPC impairs long-term fear memory for the context. $(A)$ Schematic drawing of the experimental design for delay fear conditioning indicating the timeline of the experimental manipulations $(n=6$ for chondroitinase + hyaluronidase, $n=8$ for vehicle). (B) The percentage of time spent freezing during the presentation of the CS across three trials of fear conditioning training. (C) Forty-eight hours after training, the percentage of time a rat spent freezing while exposed to either the training context (contextual fear) or during the CS. $\left({ }^{*}\right) P \leq 0.05$. (D) The percentage freezing during the 3-min context exposure when broken down into 30 -sec segments demonstrated significantly less freezing throughout the entire 3 min of testing for the enzyme-treated animals. $\left(^{*}\right) P \leq 0.05$ by a mixed-model ANOVA. All data are expressed as mean \pm S.E.M. 
memory was impaired. Further analysis revealed that animals treated with chondroitinase + hyaluronidase froze significantly less than vehicle-treated controls throughout the entire 3-min contextual testing period $\left(F_{(1,12)}=29.39, P=0.001\right)$ (Fig. 2D).

\section{Intra-hippocampal chondroitinase + hyaluronidase infusion impairs long-term fear memory for trace fear}

As hippocampal plasticity is also required for trace fear memory storage, we examined the consequences of PNN disruption using this paradigm. Groups of animals received bilateral infusions of either chondroitinase + hyaluronidase $(n=10)$ or vehicle $(n=9)$, then $3 \mathrm{~h}$ later were trained in the trace fear conditioning task (Fig. 3A). Both groups learned the task, as demonstrated by increased freezing during the tone $\left(F_{(6,102)}=45.664, P<0.001\right)$ (Fig. 3B) and trace period $\left(F_{(6,102)}=56.442, P<0.001\right)$ (Fig. 3C). However, there was no group difference in acquisition $(P>$ 0.05). As observed in delayed fear conditioning, long-term contextual memory was significantly impaired in the chondroitinase + hyaluronidase-treated rats compared to vehicle-treated controls when tested $48 \mathrm{~h}$ later (vehicle, $29.44 \% \pm 3.47 \%$; chondroitinase + hyaluronidase, $7.67 \% \pm 2.49 \%, P<0.001$ ) (Fig. 3D). When tested for CS and trace memory, animals with disrupted hippocampal PNN demonstrated significantly less freezing during both the presentation of the CS (vehicle, $42.56 \% \pm 2.69 \%$; chondroitinase + hyaluronidase, $\quad 19.98 \% \pm 2.37 \%, \quad P<0.001)$ and during the trace period (vehicle, $38.06 \% \pm 1.99 \%$; chondroitinase + hyaluronidase, $13.75 \% \pm 3.08 \%, P<0.001$ ) (Fig. 3E). Following the completion of the memory testing, animals were euthanized and the location of the infusions sites was determined by cresyl violet staining of brain sections (Fig. 3F). All animals in the delay and trace fear studies had infusion sites within the dHPC (Fig. 3F).

\section{Removal of mPFC PNN impairs the retention of long-term trace fear}

In order to test the consequence of removing the PNNs in the $\mathrm{mPFC}$ on trace fear conditioning, animals received bilateral infu- sions of chondroitinase + hyaluronidase $(n=12)$ or vehicle $(n=$ 12). Three hours later, rats were trained in the trace fear conditioning task (Fig. 4A). Although the rate at which the enzyme-infused animals acquired CS-elicited fear was significantly slower than that observed in vehicle-treated animals $\left(F_{(1,22)}=8.873, P=\right.$ 0.007 ) (Fig. 4B), both groups demonstrated equivalent freezing behaviors by the end of training. No difference was observed in the rate of learning when freezing during the trace period was assessed $\left(F_{(1,22)}=1.733, P=0.202\right)$ (Fig. 4 C).

Figure 4D shows that removal of the PNN in the mPFC had no effect on long-term contextual fear memory (vehicle, $29.5 \% \pm$ 4.67\%; chondroitinase + hyaluronidase, $22.33 \% \pm 6.02 \%, P=$ 0.357). When tested for CS-elicited fear, the chondroitinase + hyaluronidase-treated rats froze significantly less during the tone presentations than did vehicle-treated controls (vehicle, $26.40 \% \pm 4.37 \%$; chondroitinase + hyaluronidase, $13.96 \% \pm$ $2.26 \%, P=0.019)$. Freezing during the trace period was also significantly reduced following $\mathrm{mPFC}$ PNN removal (vehicle, $39.79 \% \pm 6.69 \%$; chondroitinase + hyaluronidase, $19.46 \% \pm$ $4.64 \%, P=0.021$ ) (Fig. 4E). This difference was specific to the trace period, as no difference was observed when freezing was measured $30 \mathrm{sec}$ immediately prior to CS presentation (pre-CS) $(P>0.05)$ (Fig. 4E). When the amount of freezing during the trace period was compared to the amount of freezing during the 30 -sec pre-CS period within a group, only vehicle-treated animals demonstrated an increased amount of freezing during the trace period $(P<0.05)$. This suggests that while vehicle-treated animals were able to distinguish between the trace period and pre-CS period, enzyme-treated animals failed to remember the significance of the trace period.

\section{Removal of mPFC PNN impairs cue-specific fear following delayed conditioning}

Although previous lesion studies have demonstrated that the $\mathrm{mPFC}$ is not required for delayed conditioning, a number of studies have found increased neuronal activity in the mPFC during the presentation of the CS (Baeg et al. 2001; Gilmartin and McEchron
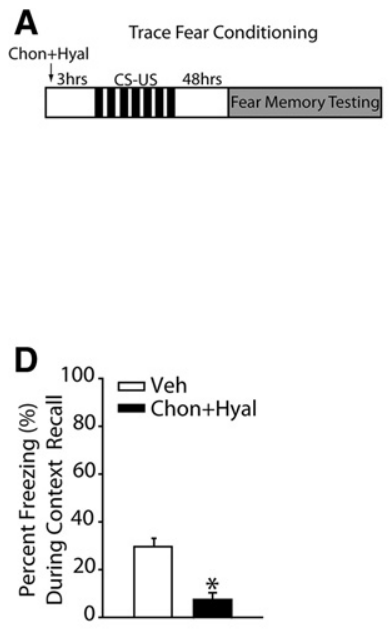
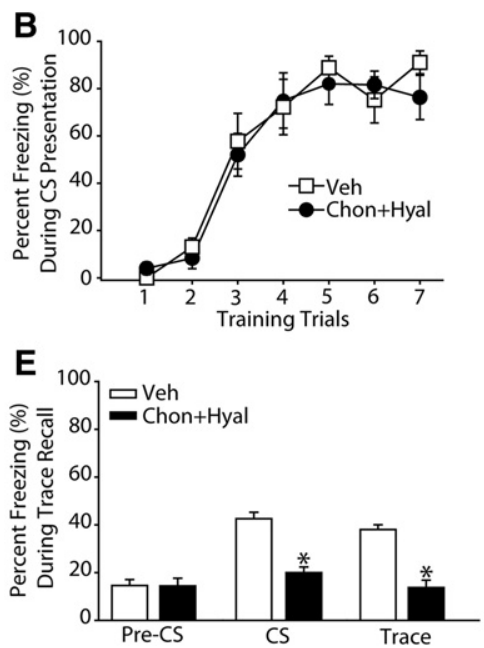

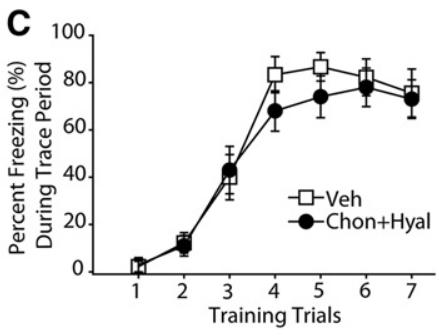

$\mathbf{F}$

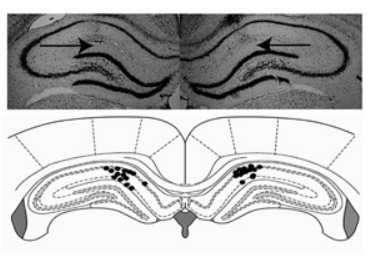

Figure 3. $\mathrm{dHPC}$ infusion of chondroitinase (Chon) + hyaluronidase (Hyal) impairs long-term trace memory. (A) Schematic drawing of the experimental design for trace fear conditioning indicating the timeline of the experimental manipulations $(n=10$ for chondroitinase + hyaluronidase, $n=9$ for vehicle). (B) The percentage of time spent freezing during the presentation of the CS. (C) The percentage of time spent freezing during the trace period during training. $(D)$ The percentage of time animals spent freezing during exposure to the training context $48 \mathrm{~h}$ following training. $(*) P \leq$ 0.05 . (E) The percentage of time animals spent freezing to the pre-CS, CS, and trace period in a novel context. $\left(^{*}\right) P \leq 0.05$. $(F)$ A representative photograph of a coronal section stained with cresyl violet with terminal sites of intra-hippocampal infusions indicated by arrows. Summary of nonredundant infusion sites for delay and trace fear experiments indicated by filled circles (illustration modified from Paxinos and Watson [2007], with permission from Elsevier $($ 2007). All data are expressed as mean \pm S.E.M. 

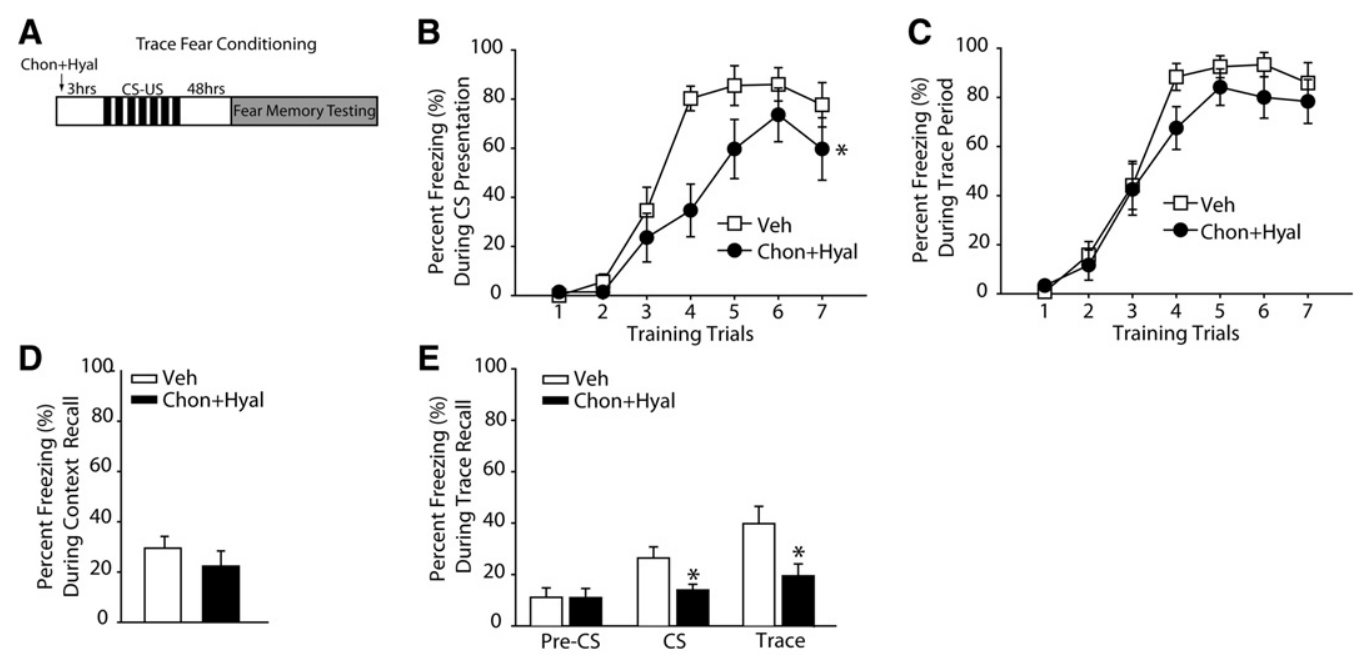

Figure 4. Removal of the PNN in the mPFC impairs trace fear memory. $(A)$ Schematic drawing of the experimental design for trace fear conditioning indicating the timeline of the experimental manipulations ( $n=12$ for chondroitinase + hyaluronidase, $n=12$ for vehicle). (B) The percentage of time spent freezing during the presentation of the CS. $\left(^{*}\right) P \leq 0.05$ by a mixed-model ANOVA. $(C)$ The percentage of time spent freezing during the trace period throughout training. $(D)$ The percentage of time animals spent freezing while exposed to the training context $48 \mathrm{~h}$ later. $(E)$ The percentage of time animals spent freezing during the pre-CS, CS, and the trace periods tested in a novel context. $\left(^{*}\right) P \leq 0.05$. All data are expressed as mean \pm S.E.M.

2005; Laviolette et al. 2005; Burgos-Robles et al. 2009). As we observed that PNN removal impaired cue-elicited fear following trace conditioning, we questioned if a similar impairment would be seen following delayed fear conditioning. Animals received either bilateral infusions of both chondroitinase + hyaluronidase $(n=10)$ or vehicle $(n=10)$ into the MPFC and were trained in delay fear conditioning $3 \mathrm{~h}$ later (Fig. 5A). Removal of the mPFC PNNs had no effect on acquisition as both groups expressed fear during the tone presentations $\left(F_{(1,18)}=0.381, P=0.545\right)$ (Fig. 5B). Although intra-mPFC infusions of chondroitinase + hyaluronidase had no effect on contextual memory (vehicle, $52.50 \% \pm 5.15$; chondroitinase + hyaluronidase, $53.30 \% \pm 5.13$, $P=0.914)$, long-term memory for the CS was disrupted in enzyme-treated animals (vehicle, $63.20 \% \pm 9.03$; chondroitinase + hyaluronidase, $15.80 \pm 3.24, P<0.001$ ) (Fig. 5C). Further analysis of freezing using 30-sec bins revealed that the treated animals froze significantly less throughout the entire 3 min of testing (Fig. 5D). Following the completion of behavioral testing, rats were euthanized and injection sites were examined on cresyl violet stained brain sections. All of the infusions were located within the MPFC for both the trace and delay fear behavioral studies (Fig. 5E).

\section{Discussion}

In the present study, we examined the role of hippocampal and mPFC PNN in long-term memory storage using both delayed and trace fear conditioning paradigms. Our results revealed three key findings: (1) disruption of the PNN in the hippocampus, but not in the mPFC, impairs context-specific memory, (2) trace fear memory is impaired by removal of PNN in either the hippocampus or in the MPFC, and (3) PNN removal in the mPFC disrupts CS-elicited fear memory in both trace and delayed fear conditioning.

Prior studies have shown that the sensory and motor cortices have high levels of PNN whereas higher association areas have relatively less PNN expression (Hockfield and McKay 1983; Hendry et al. 1988; McGuire et al. 1989; Bruckner et al. 1994,
1999, 2008; Hausen et al. 1996). As it is thought that PNNs stabilize synaptic connectivity, it has been suggested that areas with high PNN levels may be less plastic (Gati et al. 2010; Bartus et al. 2012; McRae and Porter 2012). We observed that the CA3 subfield of the hippocampus had the greatest amount of WFA staining, with less intense staining observed in other hippocampal subfields, particularly CA1. These findings are in agreement with previous reports by Bruckner et al. (2003), and suggest that the CA1 subfield of the hippocampus can readily undergo plastic changes during training in order to store learned information (Tsien et al. 1996; Blum et al. 1999).

Previous research has shown that lesions of the hippocampus impair contextual fear in both delayed and trace fear conditioning tasks (Weiss et al. 1999; Esclassan et al. 2009). Although it has been demonstrated that infusion of hyaluronidase into the hippocampus impairs contextual fear memory (Kochlamazashvili et al. 2010), the consequences of PNN disruption on trace fear memory had not been examined. Our results show that disruption of the PNN in the hippocampus does not influence the acquisition of trace fear conditioning, but impairs the memory for both the context in which the training took place, as well as for the trace period. Although the present study cannot delineate the contribution of the individual hippocampal subfields to the memory impairment we observed, recent electrophysiological recordings from the CA1 subfield have shown that during recall of a trace fear memory there is increased neural activity that peaks prior to when the shock would be expected (Chen et al. 2009; Song et al. 2012). Further, this increase in neuronal activity is observed when the animal is placed back in the original training context in the absence of the tone (Chen et al. 2009). Taken together, these data suggest that although the CA1 subfield has proportionally less PNN than other areas of the hippocampus, disruption of the PNN in this subfield likely contributed to the memory impairments we observed.

In addition to the hippocampus, the mPFC has been implicated in trace fear conditioning (Kronforst-Collins and Disterhoft 1998; Weible et al. 2000; McLaughlin et al. 2002). For example, McLaughlin et al. (2002) found that lesions of the mPFC disrupt trace eyeblink conditioning, but had no impact upon 


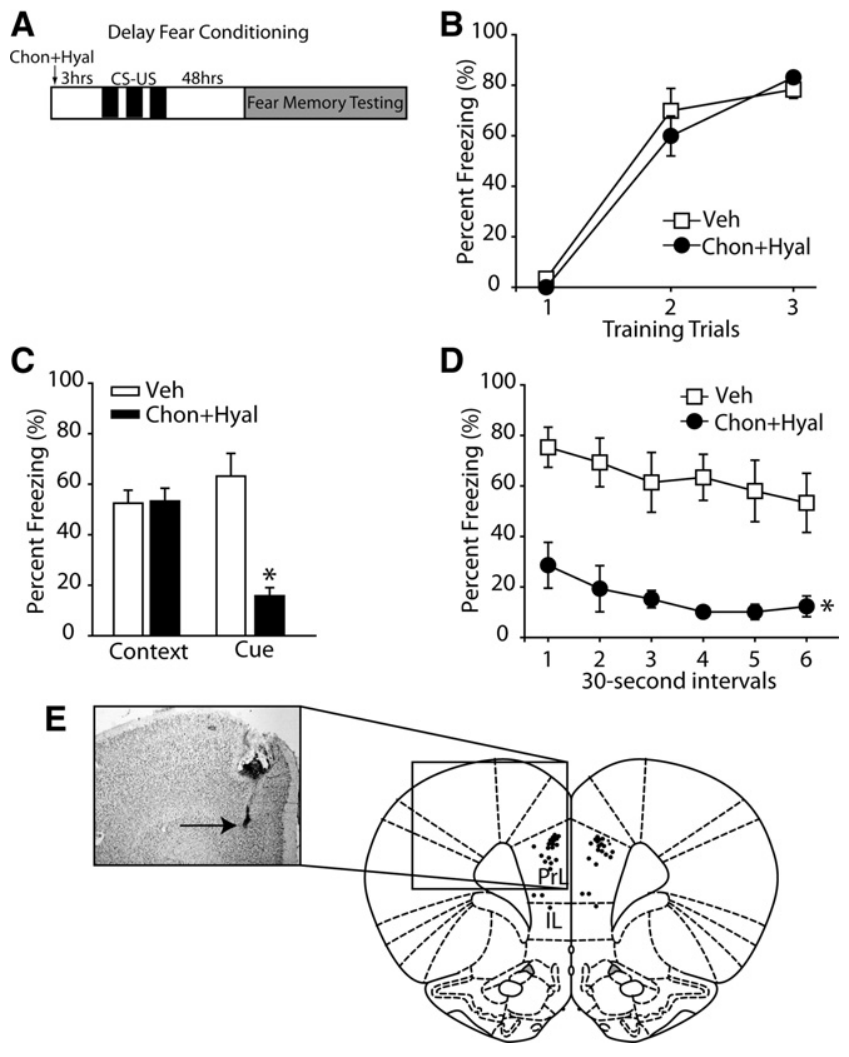

Figure 5. Long-term memory for the $\mathrm{CS}$ is impaired following PNN removal in the mPFC. (A) Schematic drawing of the experimental design for delay fear conditioning indicating the timeline of the experimental manipulations ( $n=10$ for chondroitinase + hyaluronidase, $n=$ 10 for vehicle). (B) The percentage of time spent freezing during the presentation of the CS across three trials of fear conditioning training. (C) The percentage of time spent freezing while exposed to either the training context (contextual fear) or during the CS $48 \mathrm{~h}$ after training. $\left(^{*}\right) P \leq$ 0.05. (D) During the 3-min cue exposure, when broken down into 30 -sec segments, chondroitinase + hyaluronidase-treated animals demonstrated significantly less freezing throughout the entire $3 \mathrm{~min}$ of testing. $(*) P \leq 0.05$ by a mixed-model ANOVA. (E) Representative photomicrograph of a coronal section stained with cresyl violet showing an infusion tract in the mPFC. Summary data showing the location of the nonredundant infusion sites for delay and trace fear experiments (illustration modified from Paxinos and Watson [2007], with permission from Elsevier (C) 2007). Data are mean \pm S.E.M.

delay conditioning. Furthermore, studies have shown that the mPFC undergoes plastic changes at the time of training and that these changes are required for trace memory storage (Runyan et al. 2004). Consistent with these reports, we found that preconditioning disruption of the PNN in the mPFC impairs longterm trace fear memory. Interestingly, we also observed that the acquisition of cue-elicited fear was impaired by PNN removal, further supporting a role for the MPFC in CS processing. However, how acquisition was influenced by PNN removal is not yet known. When we evaluated the distribution of PNNs in the mPFC using WFA staining, we observed that the PNN exists as a network with higher staining surrounding parvalbumin-positive inhibitory neurons (Fig. 1G). This suggests that PNN removal may impact GABAergic activity and plasticity, possibly giving rise to the acquisition effects we observed. Consistent with this, it has been reported that removal of PNN increases the excitability of inhibitory basket neurons (Dityatev et al. 2007).

In addition to influencing acquisition, we found that PNN removal in the mPFC impaired CS-elicited fear memory following both trace and delay conditioning paradigms. Although previous studies have reported that lesions of the mPFC do not disrupt delayed fear conditioning (Gewirtz et al. 1997; McLaughlin et al. 2002), recent electrophysiological recordings have shown that the activity of subsets of neurons within the MPFC increases in response to cue presentation during acquisition and recall of conditioned fear (Baeg et al. 2001; Gilmartin and McEchron 2005; Laviolette et al. 2005; Burgos-Robles et al. 2009). Our finding that PNN removal impairs cue-elicited fear memory further implicates this structure in the storage of cue-elicited fear. In addition, these findings also show that information storage occurs both in the hippocampus and in the MPFC as a direct result of training, and that the stored information in both these structures is required for the appropriate behavioral response to fearful stimuli.

\section{Materials and Methods}

\section{Materials}

Male Long Evans rats (250-275 g) were purchased from Harlan (Indianapolis). Protease free $\mathrm{ABC}$ chondroitinase was purchased from Seikagaku Corporation and hyaluronidase was bought from Calibochem. The NeuN antibody was purchased from Millipore, WFA was obtained from Sigma Aldrich, and parvalbumin antibody was purchased from Thermo Fisher Scientific.

\section{Drug preparation and administration}

All protocols involving the use of animals are in compliance with NIH's Guide for The Care and Use of Laboratory Animals and approved by The Institutional Animal Care and Use Committee. Rats were anesthetized using $4 \%$ isoflurane with a 2:1 $\mathrm{N}_{2} \mathrm{O}: \mathrm{O}_{2}$ mixture and then maintained with a $2 \%$ isoflurane/2:1 $\mathrm{N}_{2} \mathrm{O}: \mathrm{O}_{2}$ mixture via a facemask. Bilateral guide cannulae, aimed at the dHPC (AP $-3.3 \mathrm{~mm}, \mathrm{~L} \pm 2.0 \mathrm{~mm}$ from bregma, and $\mathrm{V}-2.0 \mathrm{~mm}$ from the dura) or prefrontal cortex (PFC) (AP $+3.2 \mathrm{~mm}, \mathrm{~L} \pm 0.8 \mathrm{~mm}$ from bregma, and $\mathrm{V}-2.5 \mathrm{~mm}$ from the dura), were implanted. The rats were then allowed to recover in their home cages for 10-12 d. For intra-hippocampal infusions, the injection cannulae extended $1.75 \mathrm{~mm}$ beyond the tips of the guides, yielding a total depth of $3.75 \mathrm{~mm}$ below the dura. For intra-mPFC infusions, the injection cannulae extended $1.5 \mathrm{~mm}$ beyond the tips of the guides, yielding a total depth of $4.0 \mathrm{~mm}$ below the dura. A working solution of $\mathrm{ABC}$ chondroitinase was prepared by dissolving $2 \mathrm{U}$ in sterile saline for a final injection concentration of $25 \mathrm{U} / \mathrm{mL}$. Hyaluronidase was dissolved in sterile saline for a final concentration of $0.70 \mu \mathrm{g} / \mu \mathrm{L}$. The chondroitinase + hyaluronidase working solution was prepared as follows. The hyaluronidase was initially diluted in sterile saline then added to $2 \mathrm{U}$ chondroitinase. All infusions $(1 \mu \mathrm{L} /$ hippocampus or $0.7 \mu \mathrm{L} / \mathrm{mPFC}$ of either drug or vehicle) were performed in freely moving animals at a rate of 0.25 $\mu \mathrm{L} / \mathrm{min}$ using a dual syringe infusion pump (Stoelting) followed by a 2-min waiting period. The use of these concentrations was guided by previous studies using these enzymes to disrupt HA and CSPG, respectively (Tona and Bignami 1993; Nakamura et al. 2009).

\section{Immunohistochemistry}

The influence of chondroitinase and/or hyaluronidase infusions on the disruption of the PNN was assessed in a separate group of rats than those used for behavioral testing. Rats were anesthetized as previously stated and chondroitinase and/or hyaluronidase was infused into one hippocampus (or mPFC), while an equal volume of vehicle was simultaneously infused into the contralateral hippocampus (or mPFC) of the same animal. The same dose of chondroitinase and/or hyaluronidase as used for the behavioral studies was utilized. Three hours later, rats were deeply anesthetized with sodium pentobarbital $(100 \mathrm{mg} / \mathrm{kg})$ and transcardially perfused with PBS followed by $4 \%$ paraformaldehyde. Brains were removed, cryoprotected in 30\% sucrose (in PBS), and sectioned into $40-\mu \mathrm{m}$ thick slices using a cryostat. Free-floating slices were incubated 
overnight in primary antibodies $(0.5-1.0 \mu \mathrm{g} / \mathrm{mL})$ containing $0.1 \%$ triton $X-100$ in PBS containing $2 \%$ BSA and $2 \%$ normal goat serum. After extensive washing, immunoreactivity was detected using species-specific secondary antibodies coupled to Alexafluors. Strepavidin conjugated Alexafluros was used for WFA staining. Sections were then mounted on slides and viewed using a Zeiss Axiovert microscope. Photomicrographs were taken of the prefrontal cortex and the dHPC.

\section{Fear conditioning}

All behavioral tests were performed by an investigator who was blind to the treatment groups. For delay fear conditioning, animals were placed in the training context (Habitest Unit, Coulbourne Instruments) and given a 120 -sec familiarization period. Fear conditioning trials began with a 10 -sec tone (CS) which co-terminated with a 2 -sec 1.0 -mA foot shock (US). Animals received three CS-US paired training trials, separated by a 120 -sec inter-trial interval (ITI). Forty-eight hours following fear conditioning training, each animal was tested for long-term contextual fear memory by placing it back in the original training chamber and monitoring freezing behavior every $2 \mathrm{sec}$ for a total of 3 min. In order to test long-term memory for the CS, animals were placed in a novel chamber for a 120 -sec habituation period, followed by a single 3-min tone presentation. Animals were monitored for freezing behavior during the exploratory period in the novel chamber as well as during the 3-min cue presentation.

For trace fear conditioning, rats were placed in the training context for $120 \mathrm{sec}$ (Habitest Unit, Coulbourne Instruments). Rats were then given seven training trials. Each trial consisted of a 10 -sec tone followed by a 20 -sec trace period. At the end of the trace period, animals received a $0.5-\mathrm{sec}, 0.5-\mathrm{mA}$ foot shock. Each trial was separated by an ITI that varied from 1 to $4 \mathrm{~min}$ in length. This was done in order to prevent the ITI from serving as a cue for the presentation of the US. The amount of freezing was measured $30 \mathrm{sec}$ prior to the presentation of the tone (pre-CS period), during the presentation of the tone (CS period), and during the trace period (trace period freezing). Rats were tested for contextual and cue memory $48 \mathrm{~h}$ following training. During contextual fear memory testing, rats were placed back in the original training context for $3 \mathrm{~min}$ and freezing behavior was monitored. Animals were then placed in a novel context for 120 sec and given four presentations of the CS without the US in order to test for the memory. Freezing behavior during the tone and trace period was measured. Freezing behavior is defined as the absence of all movement, excluding movement caused by respiration, and is used as a behavioral means of measuring fear.

\section{Verification of cannula placement}

Following the completion of behavioral testing, rats were euthanized by a $\mathrm{CO}_{2}$ overdose. The tissue was then removed and postfixed in $4 \%$ paraformaldehyde for $24-48 \mathrm{~h}$ and then cryoprotected in $30 \%$ sucrose. The tissue was sliced into $40-\mu \mathrm{m}$ sections and sections containing the infusion site were mounted onto slides, stained with cresyl violet, and subsequently analyzed for correct placement of the cannula.

\section{Statistical analysis}

For evaluation of behavioral data, repeated measures analyses of variance (two-way or one-way as appropriate) were used. A Holm-Sidak method for multiple comparisons post-hoc tests was used to determine data points with significant differences. Student's $t$-tests were utilized to determine statistical differences between control and experimental groups during retention testing. Data were considered significant at $P \leq 0.05$ and presented as mean \pm standard error of the mean.

\section{Acknowledgments}

We thank Melanie Moody, Christina Nelson, and Jing Zhao for their assistance with the cannulations and Min Zhang for his tech- nical assistance. The work performed in the authors' laboratories was made possible by grants from NIH (MH072933, NS053588).

\section{References}

Alpár A, Gärtner U, Härtig W, Brückner G. 2006. Distribution of pyramidal cells associated with perineuronal nets in the neocortex of rat. Brain Res 1120: $13-22$.

Baeg EH, Kim YB, Jang J, Kim HT, Mook-Jung I, Jung MW. 2001. Fast spiking and regular spiking neural correlates of fear conditioning in the medial prefrontal cortex of the rat. Cereb Cortex 11: 441-451.

Bartus K, James ND, Bosch KD, Bradbury EJ. 2012. Chondroitin sulphate proteoglycans: Key modulators of spinal cord and brain plasticity. Exp Neurol 235: 5-17.

Blum S, Moore AN, Adams F, Dash PK. 1999. A mitogen-activated protein kinase cascade in the CA1/CA2 subfield of the dorsal hippocampus is essential for long-term spatial memory. J Neurosci 19: $3535-3544$.

Bruckner G, Seeger G, Brauer K, Hartig W, Kacza J, Bigl V. 1994. Cortical areas are revealed by distribution patterns of proteoglycan components and parvalbumin in the Mongolian gerbil and rat. Brain Res 658: $67-86$.

Bruckner G, Hausen D, Hartig W, Drlicek M, Arendt T, Brauer K. 1999. Cortical areas abundant in extracellular matrix chondroitin sulphate proteoglycans are less affected by cytoskeletal changes in Alzheimer's disease. Neuroscience 92: 791-805.

Bruckner G, Grosche J, Hartlage-Rubsamen M, Schmidt S, Schachner M. 2003. Region and lamina-specific distribution of extracellular matrix proteoglycans, hyaluronan and tenascin-R in the mouse hippocampal formation. J Chem Neuroanat 26: 37-50.

Bruckner G, Morawski M, Arendt T. 2008. Aggrecan-based extracellular matrix is an integral part of the human basal ganglia circuit. Neuroscience 151: 489-504.

Bukalo O, Schachner M, Dityatev A. 2001. Modification of extracellular matrix by enzymatic removal of chondroitin sulfate and by lack of tenascin-R differentially affects several forms of synaptic plasticity in the hippocampus. Neuroscience 104: 359-369.

Burgos-Robles A, Vidal-Gonzalez I, Quirk GJ. 2009. Sustained conditioned responses in prelimbic prefrontal neurons are correlated with fear expression and extinction failure. J Neurosci 29: 8474-8482.

Chen G, Wang LP, Tsien JZ. 2009. Neural population-level memory traces in the mouse hippocampus. PLoS One 4: e8256.

Dash PK, Hebert AE, Runyan JD. 2004. A unified theory for systems and cellular memory consolidation. Brain Res Brain Res Rev 45: 30-37.

Dityatev A, Bruckner G, Dityateva G, Grosche J, Kleene R, Schachner M. 2007. Activity-dependent formation and functions of chondroitin sulfate-rich extracellular matrix of perineuronal nets. Dev Neurobiol 67: $570-588$.

Esclassan F, Coutureau E, Di SG, Marchand AR. 2009. Differential contribution of dorsal and ventral hippocampus to trace and delay fear conditioning. Hippocampus 19: 33-44.

Gati G, Morawski M, Lendvai D, Matthews RT, Jager C, Zachar G, Arendt T, Alpar A. 2010. Chondroitin sulphate proteoglycan-based perineuronal net establishment is largely activity-independent in chick visual system. J Chem Neuroanat 40: 243-247.

Gewirtz JC, Falls WA, Davis M. 1997. Normal conditioned inhibition and extinction of freezing and fear-potentiated startle following electrolytic lesions of medial prefrontal cortex in rats. Behav Neurosci 111: $712-726$.

Gilmartin MR, McEchron MD. 2005. Single neurons in the dentate gyrus and CA1 of the hippocampus exhibit inverse patterns of encoding during trace fear conditioning. Behav Neurosci 119: 164-179.

Gogolla N, Caroni P, Luthi A, Herry C. 2009. Perineuronal nets protect fear memories from erasure. Science 325: 1258-1261.

Hartig W, Brauer K, Bruckner G. 1992. Wisteria floribunda agglutinin-labelled nets surround parvalbumin-containing neurons. Neuroreport 3: 869-872.

Hausen D, Bruckner G, Drlicek M, Hartig W, Brauer K, Bigl V. 1996. Pyramidal cells ensheathed by perineuronal nets in human motor and somatosensory cortex. Neuroreport 7: 1725-1729.

Hendry SH, Jones EG, Hockfield S, McKay RD. 1988. Neuronal populations stained with the monoclonal antibody Cat-301 in the mammalian cerebral cortex and thalamus. J Neurosci 8: 518-542.

Hockfield S, McKay RD. 1983. A surface antigen expressed by a subset of neurons in the vertebrate central nervous system. Proc Natl Acad Sci 80: $5758-5761$.

Kochlamazashvili G, Henneberger C, Bukalo O, Dvoretskova E, Senkov O, Lievens PM, Westenbroek R, Engel AK, Catterall WA, Rusakov DA, et al. 2010. The extracellular matrix molecule hyaluronic acid regulates hippocampal synaptic plasticity by modulating postsynaptic L-type $\mathrm{Ca}^{2+}$ channels. Neuron 67: 116-128. 
Kronforst-Collins MA, Disterhoft JF. 1998. Lesions of the caudal area of rabbit medial prefrontal cortex impair trace eyeblink conditioning. Neurobiol Learn Mem 69: 147-162.

Laviolette SR, Lipski WJ, Grace AA. 2005. A subpopulation of neurons in the medial prefrontal cortex encodes emotional learning with burst and frequency codes through a dopamine D4 receptor-dependent basolateral amygdala input. J Neurosci 25: 6066-6075.

McGuire PK, Hockfield S, Goldman-Rakic PS. 1989. Distribution of cat-301 immunoreactivity in the frontal and parietal lobes of the macaque monkey. J Comp Neurol 288: 280-296.

McLaughlin J, Skaggs H, Churchwell J, Powell DA. 2002. Medial prefrontal cortex and Pavlovian conditioning: Trace versus delay conditioning. Behav Neurosci 116: $37-47$.

McRae PA, Porter BE. 2012. The perineuronal net component of the extracellular matrix in plasticity and epilepsy. Neurochem Int 61: 963-972.

Nakamura M, Nakano K, Morita S, Nakashima T, Oohira A, Miyata S. 2009. Expression of chondroitin sulfate proteoglycans in barrel field of mouse and rat somatosensory cortex. Brain Res 1252: $117-129$.

Nowicka D, Soulsby S, Skangiel-Kramska J, Glazewski S. 2009. Parvalbumin-containing neurons, perineuronal nets and experience-dependent plasticity in murine barrel cortex. Eur J Neurosci 30: $2053-2063$.

Paxinos G, Watson C. 2007. The rat brain in stereotaxic coordinates, 6th ed. Elsevier, New York.

Pizzorusso T, Medini P, Berardi N, Chierzi S, Fawcett JW, Maffei L. 2002. Reactivation of ocular dominance plasticity in the adult visual cortex. Science 298: $1248-1251$.
Pizzorusso T, Medini P, Landi S, Baldini S, Berardi N, Maffei L. 2006. Structural and functional recovery from early monocular deprivation in adult rats. Proc Natl Acad Sci 103: 8517-8522.

Runyan JD, Moore AN, Dash PK. 2004. A role for prefrontal cortex in memory storage for trace fear conditioning. J Neurosci 24: 1288-1295.

Saghatelyan AK, Gorissen S, Albert M, Hertlein B, Schachner M, Dityatev A. 2000. The extracellular matrix molecule tenascin-R and its HNK-1 carbohydrate modulate perisomatic inhibition and long-term potentiation in the CA1 region of the hippocampus. Eur J Neurosci 12: $3331-3342$.

Song C, Detert JA, Sehgal M, Moyer JR Jr. 2012. Trace fear conditioning enhances synaptic and intrinsic plasticity in rat hippocampus. J Neurophysiol 107: 3397-3408.

Tona A, Bignami A. 1993. Effect of hyaluronidase on brain extracellular matrix in vivo and optic nerve regeneration. J Neurosci Res 36: $191-199$.

Tsien JZ, Huerta PT, Tonegawa S. 1996. The essential role of hippocampal CA1 NMDA receptor-dependent synaptic plasticity in spatial memory. Cell 87: $1327-1338$.

Weible AP, McEchron MD, Disterhoft JF. 2000. Cortical involvement in acquisition and extinction of trace eyeblink conditioning. Behav Neurosci 114: 1058-1067.

Weiss C, Bouwmeester H, Power JM, Disterhoft JF. 1999. Hippocampal lesions prevent trace eyeblink conditioning in the freely moving rat. Behav Brain Res 99: 123-132.

Received December 21, 2012; accepted in revised form February 6, 2013. 


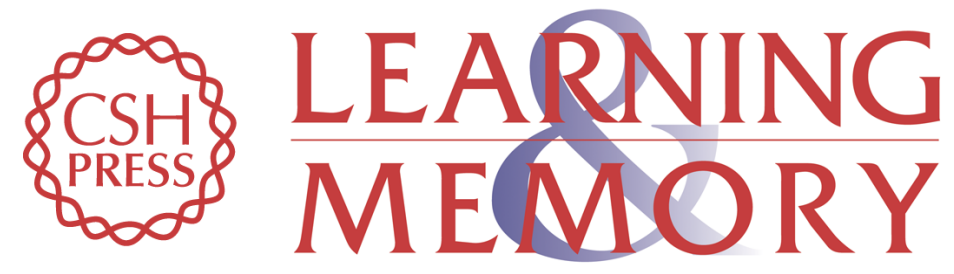

\section{Disruption of the perineuronal net in the hippocampus or medial prefrontal cortex impairs fear conditioning}

Michael J. Hylin, Sara A. Orsi, Anthony N. Moore, et al.

Learn. Mem. 2013, 20:

Access the most recent version at doi:10.1101/Im.030197.112

References This article cites 39 articles, 9 of which can be accessed free at: http://learnmem.cshlp.org/content/20/5/267.full.html\#ref-list-1

License

Email Alerting Receive free email alerts when new articles cite this article - sign up in the box at the Service top right corner of the article or click here. 